\title{
Method of the Analysis of Materials' Microstructure Based on the Fractal Analysis of Images
}

\author{
Konstantin Makarenko ${ }^{1}$ and Ekaterina Zentsova ${ }^{1}$ \\ ${ }^{1}$ Bryansk State Technical University, 50 years of October blvd., 7, Bryansk, 241035, Russian Federation
}

\begin{abstract}
The basics of metallography and modern systems used to study and analyse the structures of materials are presented. Special attention is paid to the methods of quantitative microscopy. The review of modern computer programs for analysis of image microstructures obtained from digital microscopes is given. The fundamentals of fractal analysis as a highly effective tool for calculating numerical values of parameters of geometrically complex objects are described. The analysis of the graphitized cast iron structure is provided as an example; the application of the fractal analysis method for determining such characteristics of the graphite phase as the shape of graphite inclusions and their distribution in the amount of the alloy is demonstrated. In the course of the research, different classes of cast iron have been studied. To determine the shape of graphite inclusions it was suggested to use fractal dimension. The nonuniformity of the distribution was estimated by such function as lacunarity. The separate stages of determining these characteristics with a specialized FracLac plugin within the ImageJ program are presented. The results obtained have shown high adequacy. In spite of positive assessments, there are shortcomings revealed in the course of the research on the application of fractal analysis methods for identifying geometrically complex dimensional and topological parameters of the graphite phase in cast iron. The ways to further improve these methods in order to solve a wide range of problems in metallography of alloys are suggested.
\end{abstract}

\section{Keywords}

Cast iron, graphite phase, microstructure, fractal analysis, parameters, shape, distribution.

\section{Introduction}

Until relatively recently, the researches of the materials' structures have been limited by a microscope and method of the optical microscopy. In metallography there have been always problem of verification of the research results. Traditionally, the operations of processing results, recognition, classification of the studied materials' structures, used to fall on a researcher, who was responsible for the accuracy of the presented verdicts [1-4]. Among the applied methods of recognition and classification of structures, the main one was a method of visual evaluation, based on the comparison of materials' real structure with reference images. In this approach, a metallographer was required to have profound knowledge in the area of forming of materials' structure. Taking into account the close relationship in the system: chemical composition $\rightarrow$ structure $\rightarrow$ properties; and a key role of material's structure in the forming of mechanical, physico-chemical, operational and a number of technological properties, the methods of quantative metallography were developed [5]. These methods are based on the usage of different size-topological parameters that allow to characterize the observed structure of the material with numerical and descriptive method. Meanwhile, in Materials Science, these researches are extremely in demand from the perspective of development of the mathematical models' impact of material's structure on its properties. Such models allow you, with established interrelations of sizetopological parameters of materials' structure and properties, to develop new alloys with unique properties.

GraphiCon 2021: 31st International Conference on Computer Graphics and Vision, September 27-30, 2021, Nizhny Novgorod, Russia EMAIL: makkon1@yandex.ru (Konstantin Makarenko); kopilka.32@mail.ru (Ekaterina Zentsova).

ORCID: 0000-0002-7195-0500 (Konstantin Makarenko); 0000-0003-1513-3271 (Ekaterina Zentsova).

(c) (i) (C) 2021 Copyright for this paper by its authors.

Use permitted under Creative Commons License Attribution 4.0 International (CC BY 4.0).

CEUR Workshop Proceedings (CEUR-WS.org) 
With the growth of digitalization and application of computer technology in Materials Science, a new direction of microscopy - a digital (computer) microscopy [6-7], based on the analysis of images of different structures with the help of a special analytical complex, has been developed. Modern analytical complexes, used in Materials Science, allow to determine of objects on the image, as: area [8], diameter, length, width and perimeter that are impossible to determine manually; all this makes these programs to be even more valuable; these complexes also allow to evaluate errors [9] and analyze "disconnected" objects.

Among all the variety of programs, used for solving tasks in Materials Science, it is possible to select, if not the most popular, then the most successful. The comparative analysis is presented in Table 1.

Table 1

Comparative analysis of programs, used for the analysis of images of materials' microstructures

\begin{tabular}{|c|c|}
\hline Program & Features \\
\hline 1 & 2 \\
\hline Photom & $\begin{array}{l}\text { Has a variety of functionality for processing and analysis of images, as well as all } \\
\text { similar programs. It is considered to be the easiest in mastering and using. Designed } \\
\text { for digital photometry. }\end{array}$ \\
\hline Avizo & $\begin{array}{l}\text { Designed for work with microstructures' images, detection of their defects and also } \\
\text { determination of the main morphological parameters. }\end{array}$ \\
\hline Optimas & $\begin{array}{l}\text { Combines a great number of different proven methods of image processing as well } \\
\text { as mathematical methods. A distinctive feature is the presence of two control } \\
\text { panels: a standard one - default control panel and custom panel - recording } \\
\text { capability till } 20 \text { macros; the panels simplify the work with the program. }\end{array}$ \\
\hline $\begin{array}{l}\text { Image Expert } \\
\text { Pro }\end{array}$ & $\begin{array}{l}\text { The aim of program to determine and define qualitative and quantative } \\
\text { characteristics, there is an ability to record techniques, to present and store the } \\
\text { results in any form: tables, histograms, images etc. there is an ability to work with } \\
\text { camera remotely and to configure the optical system of the complex. }\end{array}$ \\
\hline Video test & $\begin{array}{l}\text { Has similar features, there is a feature to distribute objects according to their } \\
\text { brightness and also to determine geometrical shapes in three- dimensional space } \\
\text { due to successful optical techniques, integrated into the program. }\end{array}$ \\
\hline Smart-eye & $\begin{array}{l}\text { An ability to determine quantative and quantative characteristics of any material, } \\
\text { the final task is a statistical processing of results and building graphical } \\
\text { dependencies }\end{array}$ \\
\hline Image J & $\begin{array}{l}\text { A program for processing and analysis of images with open source code, written by } \\
\text { National Institutes of Health [10] in the language Java, what allows to automatize } \\
\text { complex repeated actions [11]. Open source code allows to add optionals modules } \\
\text { (plugins) to non - developers. Plugins allow to broaden program functions and } \\
\text { perform visualization till to x-ray image [12]. }\end{array}$ \\
\hline Jmicro Vision & $\begin{array}{l}\text { Generally, an unremarkable program, similar to other with the same features and } \\
\text { functions. }\end{array}$ \\
\hline Endrov & $\begin{array}{l}\text { A program with an open source code for images' processing and analysis, based on } \\
\text { the language Java and mostly, on the philosophy Image J, but is trying to replace it } \\
\text { with the modern design. The program is designed as to differentiate the graphic } \\
\text { interface code from other data, modules, filters and also between each other. The } \\
\text { emphasis is on how to reduce the code redundancy and simplify the maintenance. } \\
\text { This program can be considered as a successor, a student of Image J, that during its } \\
\text { formation haven't taken a wrong turn, trying to attract attention. }\end{array}$ \\
\hline
\end{tabular}


The product combines in itself both a data library and a visualization program, meanwhile sharing everything among itself and violating backward compatibility for the design, considering that a big number of plugins without compatibility's violation lead to errors in design. Among the features we can distinguish a processing of additional measurements what is important for a serious microscopy, besides, the program has its own images format, not preventing the program from supporting other more popular applications.

Open CV The set algorithms of image processing, of computer vision and other general algorithms, built on the language $\mathrm{C} / \mathrm{C}++$, it is also being developed on other popular languages Java, Python, Matlab and others. Includes some narrowly - focused compact modules: the image processing, the mathematical modules, the modules of machine teaching, the motion tracking and the object derection on an image, the camera calibration and others.

With the appearance of the analytical complexes, the work with microstructures' images has simplified greatly, but in parallel, with the ease of work, there has appeared a possibility to solve more complex, multilevel tasks and carry out a lot of researches on the image processing and analysis. All this has required the development of new method is a method, based on the fractal analysis [13], or rather on the theory of fractals and fractal geometry [14]. With thes method, you can describe geometrically complex objects, which include graphite in cast iron, through the single parameter that is the fractal dimension.

Fractal - from the French "consisting of fragments". This is a geometrical object or a subset of space, characterized by self-similarity and the fractal dimension of which is bigger than the topological one. Within the fractal theory, you can analyze images of different microstructures of materials [15].

On the whole, the fractal analysis [16] is the method, the aim of which is to evaluate and assign to an image data set, the fractal characteristics which include the main and the most common - dimension, determining the object complexity; shape coefficient and entropy.

The fractal analysis consists of some methods [17]; the multifractal analysis, the lacunarity analysis, mass methods and the counting of boxes. The necessity to have reference patterns for interpretation and evaluation of the obtained results, unites all these types of analysis.

The purpose of the researches is a development of the method of images' analysis of the graphitized cast irons' microstructures, based on the fractal analysis, that will allow to identify a shape of graphite inclusions through one parameter - the fractal dimension.

\section{The Research Technique}

The simpliest and most common type of the fractal analysis of images is the counting of boxes, also known as the grid method.

The method is to collect data for the analysis, splitting an image with a grid into cells of gradually changing size. Data collection or scanning takes place in several stages, at each of the following stages, the cell size increase. The fractal characteristic of the image here is the fractal dimension, which is determined by the following formula:

where $\mathrm{N}-$ a number of cells with $\varepsilon$ size.

$$
D=\lim _{\varepsilon \rightarrow 0}\left(\frac{\ln N}{\ln \varepsilon}\right),
$$

The algorithm of the grid method has several scanning schemes, determining how the data will be collected, but in fact, how the grid will move during the image scanning. A traditional scheme is the fixed scanning by non - overlapping grid - a multiple grip, position, where the grid cells aren't overlapped and don't overlap the place where the have been before.

And this action is repeated until the whole area of the image is scanned. The second scheme implies scanning with a sliding grid when every grid cell overlaps its previous place moving through the image. 
This approach is often applied in the analysis of lacunarity and in the multifractal analysis. The third scheme is subsampling and local measurements used to determine local variations when the grid moves according some function connected with the section being scanned.

Thus, the suggested method is based on fractal analysis; or to be more precise, the grid method discussed above is applied. Data collection is carried out by fixed scanning of the microstructure image with non-overlapping grid. The method is based on the ImageJ program $[18,19]$ via its specialized FracLac plugin. ImageJ is a freely available, cross-platform open-source program, owing to which it can be enhanced by creating additional modules (plugins) and writing macros. The Java language increases performance. The software has got both standard image processing functions and the ability to conduct arithmetic and logical operations with images, to calculate statistical and geometric indicators, to construct histograms, graphs and other geometric dependencies. It is possible to work with any number of images simultaneously, limited only by available memory. FracLac is an additional module (plugin) for ImageJ, freely available too. The name, composed of two words 'fractal' and 'lacunarity', indicates its specifics. It is used for working with digital images, in particular, for measuring morphological features of the objects geometrically complex to describe. It deals with binary images, and, in our case, allows determining the fractal dimension in several ways.

\section{The Research Results and Their Analysis}

The microstructure images of several types of cast iron with different shapes of graphite have been used in the research; they are gray cast iron (GCI) with laminar graphite (Fig. 1, a), cast iron with vermicular graphite (CIVG) (Fig. 1, b) and two images of ductile cast iron (DCI) with spherical graphite (Fig. 1, c, d). Since binary images are necessary for the FracLac module, image binarization and different artifacts removal that could negatively affect the accuracy of the results have been carried out.

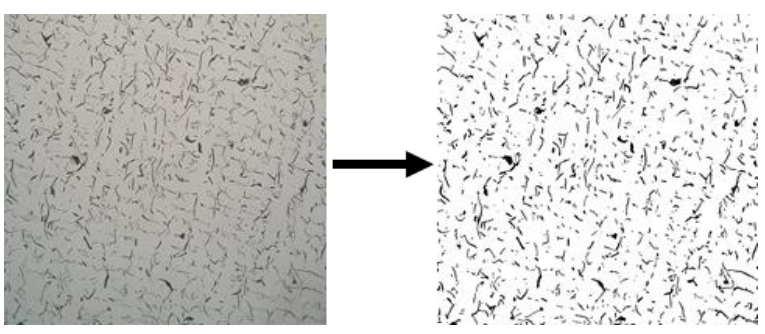

a)

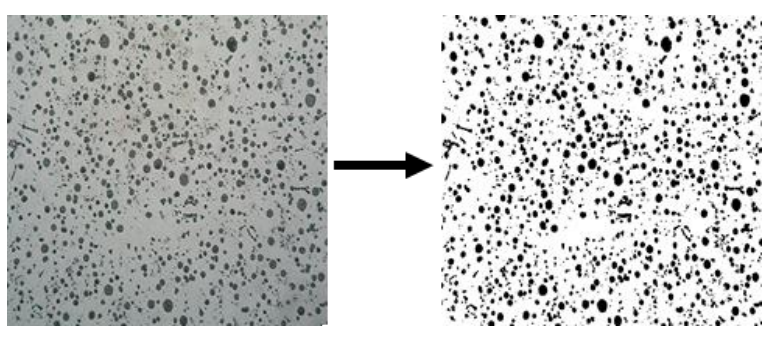

c)

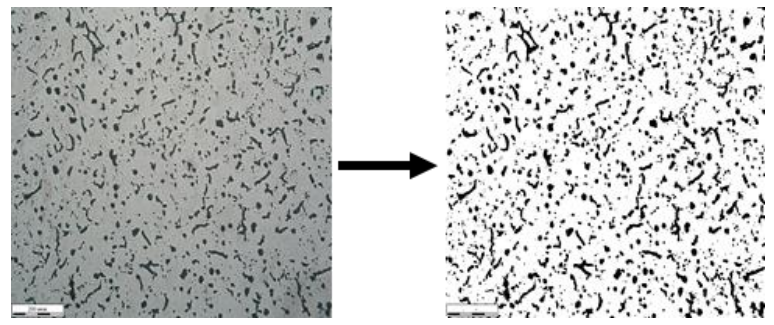

b)

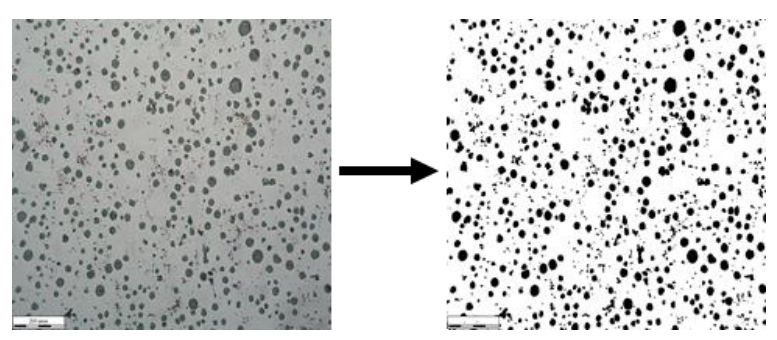

d)

Figure 1: Initial and binary images of cast iron microstructures with different graphite shapes: a) GCI with laminar graphite; b) CIVG; c) DCI with spherical graphite No. 1; d) DCI with spherical graphite No. 2

The following analysis is carried out in accordance with the algorithm of the grid method. Primarily, a range of grid block sizes is set: the minimum size is 1 pixel (minimum allowable size), the maximum one is $45 \%$ of the image (the optimal parameter; the lower value leads to the accuracy falling; the value of more than 50\% can bring to errors; there has been no difference in the values between $45 \%$ and $50 \%$ of the image). If the program chooses the number of scanning stages itself, it offers a matrix of values (Fig. 2), according to which the scanning will take place (Fig. 3), and the grid size will increase in an arithmetic progression until the progression reaches the maximum block size set, and in this case, there 
will be a fluctuating surge between next to last and the last numerical values, resulting in a sharp increase in the grid size. Along with selecting the grid size parameters, the initial values of the graphical determination of the fractal dimension and other possible parameters of the fractal image analysis of cast iron microstructures are set.

$\begin{array}{rrrrrrrrrr}1 & 5 & 9 & 13 & 17 & 21 & 25 & 29 & 33 & 37 \\ 41 & 45 & 49 & 53 & 57 & 61 & 65 & 69 & 73 & 77 \\ 81 & 85 & 89 & 93 & 97 & 101 & 105 & 109 & 113 & 117 \\ 121 & 125 & 129 & 133 & 137 & 141 & 145 & 149 & 153 & 157 \\ 161 & 165 & 169 & 173 & 177 & 181 & 185 & 189 & 193 & 197 \\ 201 & 205 & 209 & 213 & 217 & 221 & 225 & 229 & 233 & 237 \\ 241 & 245 & 249 & 253 & 257 & 261 & 265 & 269 & 273 & 277 \\ 281 & 285 & 289 & 293 & 297 & 301 & 305 & 309 & 313 & 317 \\ 321 & 325 & 329 & 333 & 337 & 341 & 345 & 349 & 353 & 357 \\ 361 & 365 & 369 & 373 & 377 & 381 & 385 & 389 & 393 & 450\end{array}$

Figure 2: Arithmetic progression of scanning (scanning matrix)

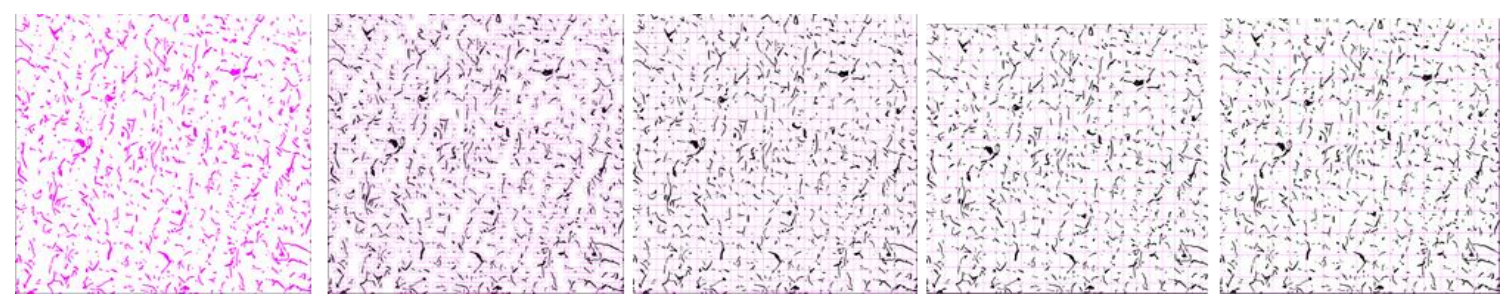

a)

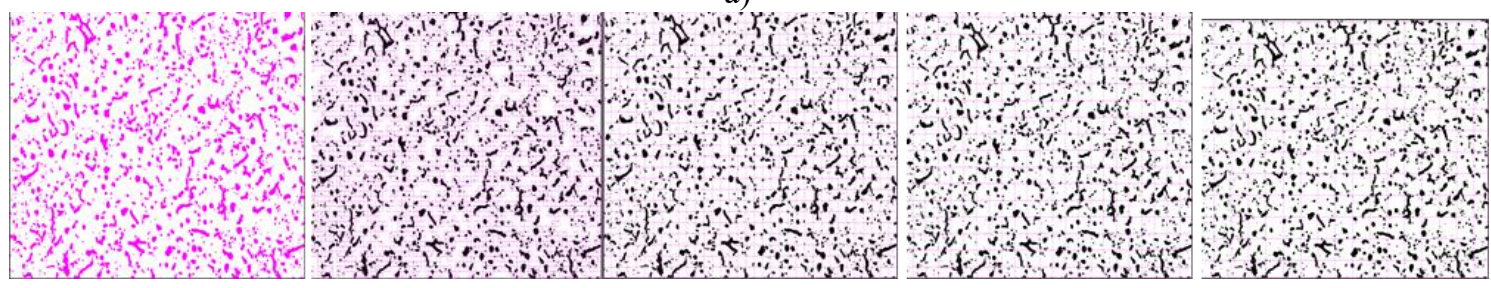

b)
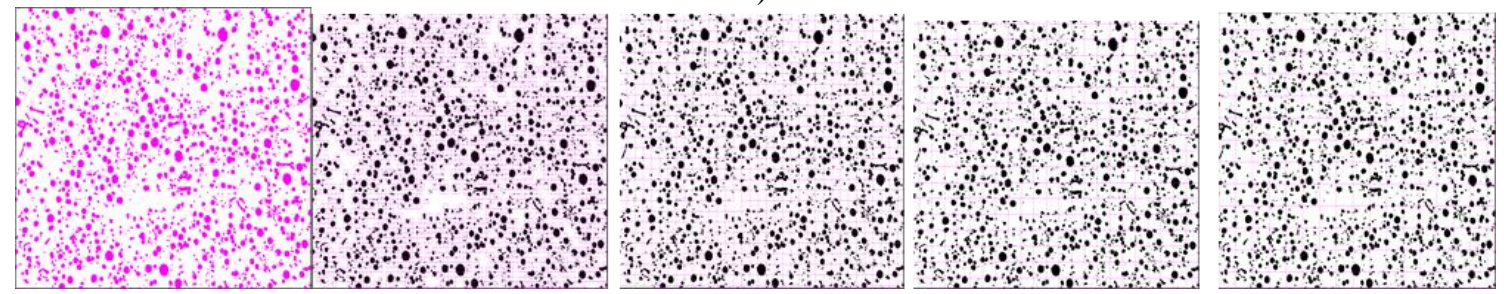

c)

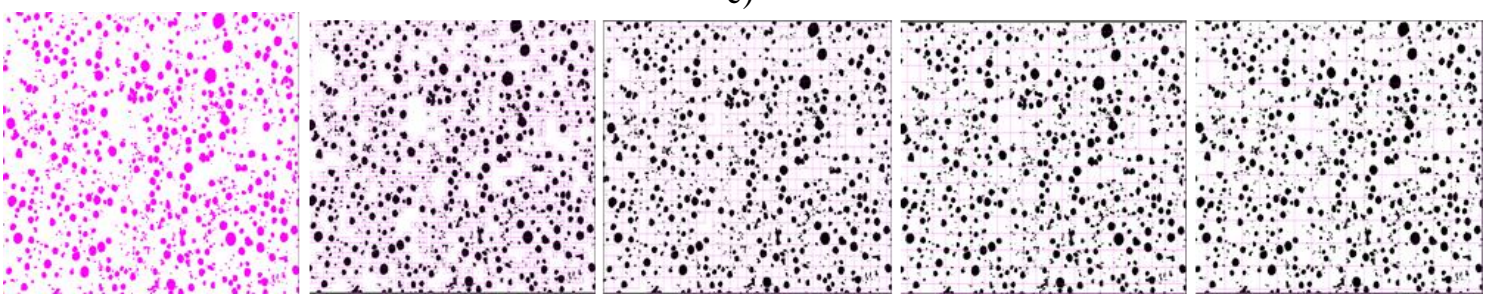

d)

Figure 3: Separate stages of image scanning of graphitized cast iron structure by the grid method: a) $\mathrm{GCl}$ with laminar graphite; b) CIVG; c) DCl with spherical graphite No. 1; d) DCl with spherical graphite No. 2

In graphs the fractal dimension is determined by the slope of the trend line, constructed by the method of least squares in the coordinates $(-\ln \varepsilon)-\ln F$ (Fig. 4). To evaluate the adequacy of the results 
obtained the coefficient of determination $\left(\mathrm{r}^{2}\right)$ is rated too. In the present research there is a high degree of correlation between the fractal dimension $(F)$ and the grid block size $(\varepsilon) \mathrm{r}^{2}=0.99$.

In addition to the fractal dimension characterizing the shape of graphite inclusions, the quantity, size and distribution can be determined in the analysis. All these parameters characterizing the graphite phase in cast iron are presented in GOST 3443-87 "Cast iron castings with graphite of different forms. Methods of structure determination", currently in force. In accordance with the methods suggested, the average size of graphite inclusions is assessed by the ratio of the total graphite areas $\left(S_{i}\right)$ to the total number of inclusions $(n)$ :

$$
A_{g r}=\frac{\sum_{i=1}^{i=n} s_{i}}{n} .
$$

The quantity of the graphite phase is calculated by the ratio of the number of black pixels $\left(\mathrm{C}_{\mathrm{gr}}\right)$ occupied by graphite to the total number of pixels of the entire image $\left(\mathrm{C}_{\mathrm{im}}\right)$ :

$$
S_{g r}=\frac{C_{g r}}{C_{i m}} 100 \% .
$$

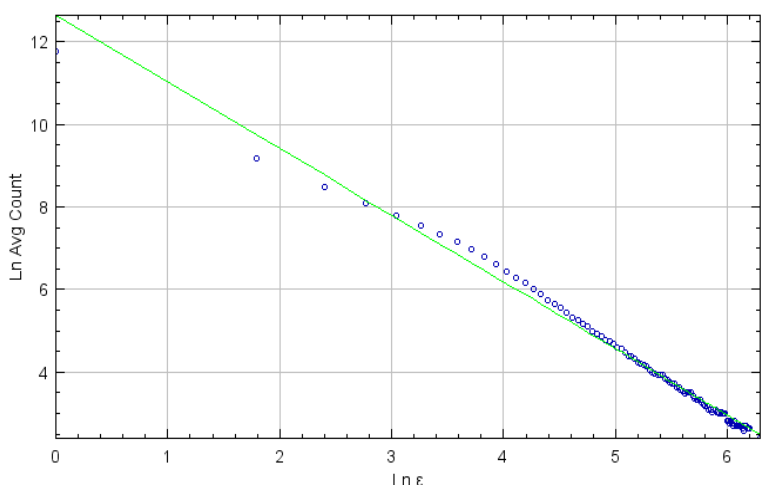

a)

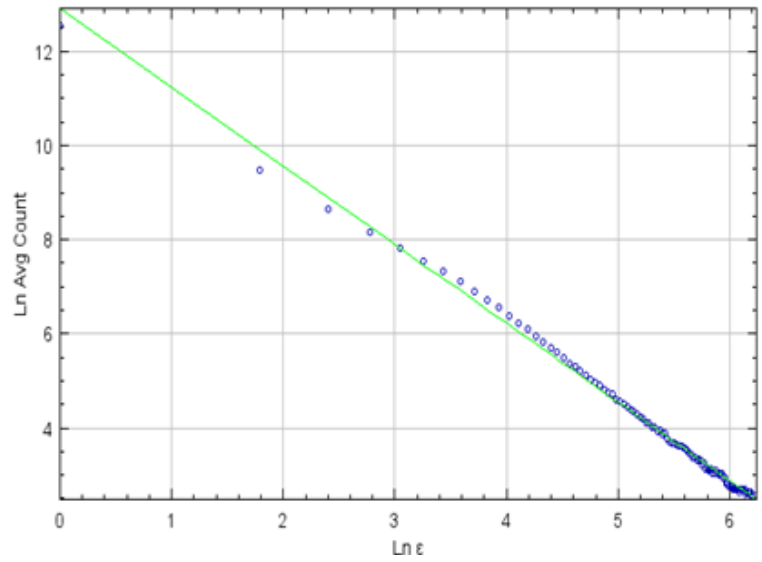

c)

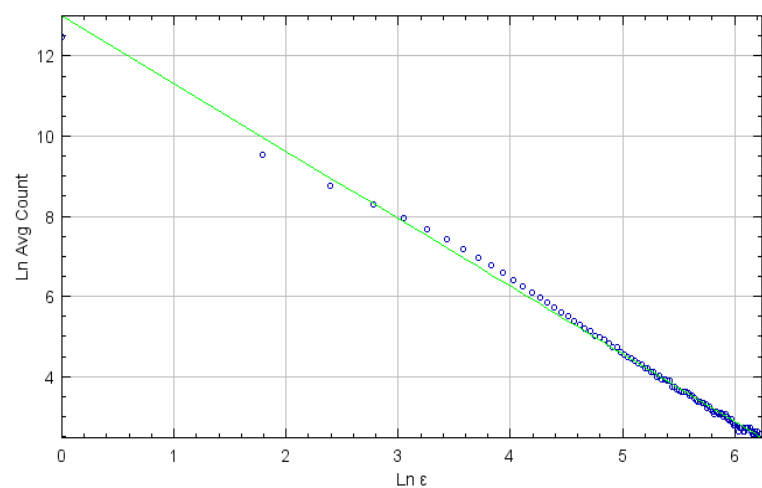

b)

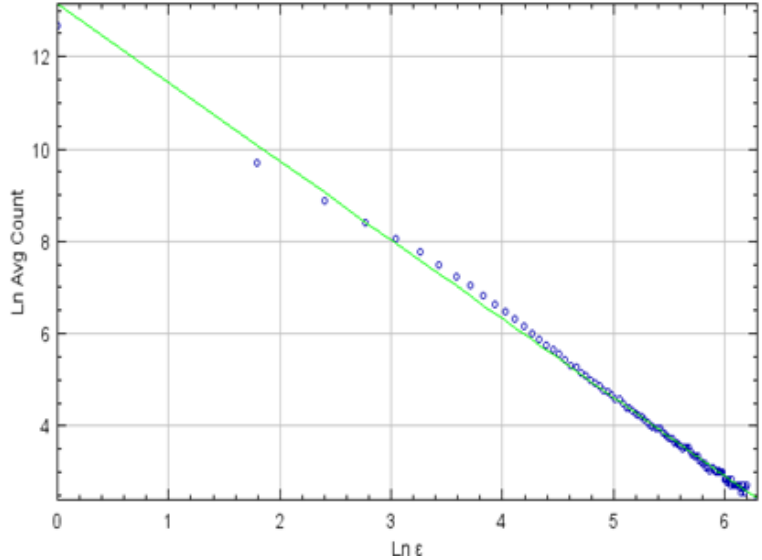

d)

Figure 4: The graphical method for determining the fractal dimension of graphite inclusions in cast iron: a) GCI with laminar graphite; b) CIVG; c) DCI with spherical graphite No. 1; d) DCI with spherical graphite No. 2

The graphite phase distribution in the amount of metal matrix of cast iron is characterized by lacunarity as a change in the image density. The lower the lacunarity is, the more lacunae there are in the image, thus implying that the graphite distribution is more uneven too. To determine the fractal dimension it is enough to analyse separate inclusions, while to estimate the distribution it is necessary to make a total analysis of the entire image. The numerical value of the lacunarity is calculated according to the following formula:

$$
\Lambda=\left(\frac{\sigma}{\mu}\right)^{2},
$$


where $\sigma$ is a standard mass deviation, $\mu$ is a mass average value of the image.

To calculate the fractal dimension it is needed to construct a graph, while to estimate the lacunarity the graphical method of least squares is used. It should be clarified, that the slope of the trend line (Fig. 5 ) is calculated by some different formula:

$$
\Lambda=\left(\frac{\sigma}{\mu}\right)^{2}+1,
$$

This formula is used to determine the slope of the trend line in order to avoid "uncertain" calculations when the image is homogeneous. An image is considered homogeneous when the number of pixels in the blocks does not change at different stages of scanning; so $\sigma=0$, and, therefore, $\Lambda=0$. In this case $\ln \Lambda=0$ too; that means that the slope of the trend line will be uncertain.

The generalized values of the graphite phase parameters for different types of cast iron are shown in Table 2. Since the reference patterns are needed to interpret and evaluate the results of the fractal analysis, an interpretation method was suggested. This is an expert estimation method based on the expert's experience and knowledge. An expert interprets and gives a judgemental estimate of the results on the basis of his/her knowledge and experience gained, and using the approaches of this method. According to the method proposed and the exact information about the microstructures, types and the graphite shapes of the cast iron studied, it can be said that the presented values of fractal dimension with the range of $+/-0.5$, as not all graphite shapes were used for the analysis, correspond to these cast iron grades and graphite shapes.

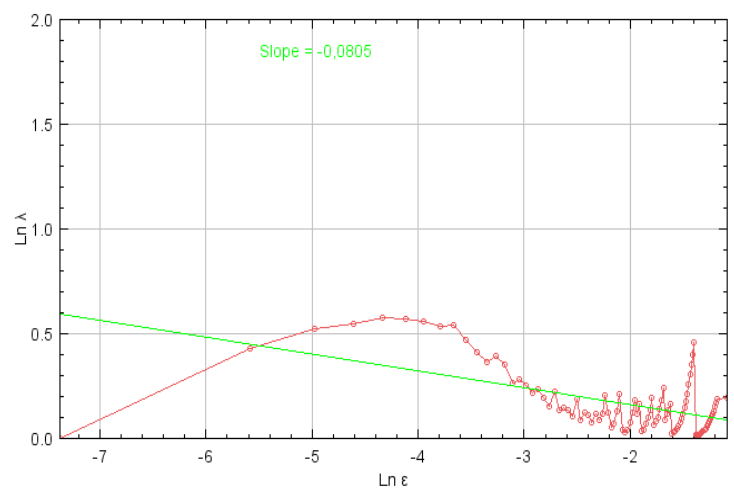

a)

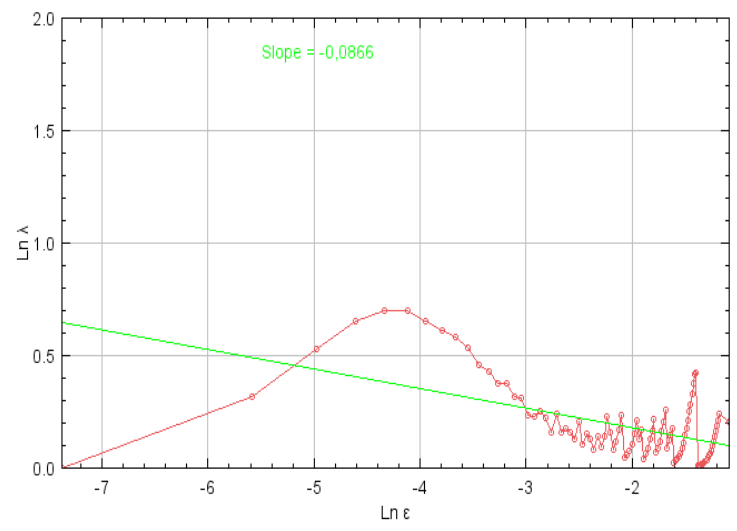

c)

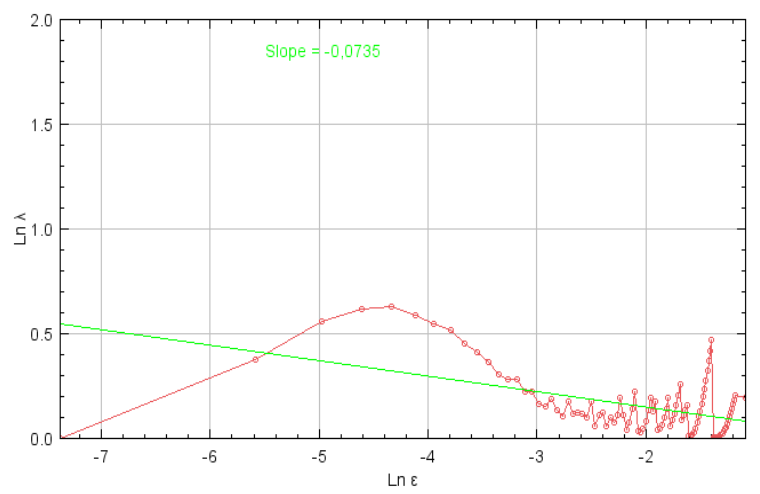

b)

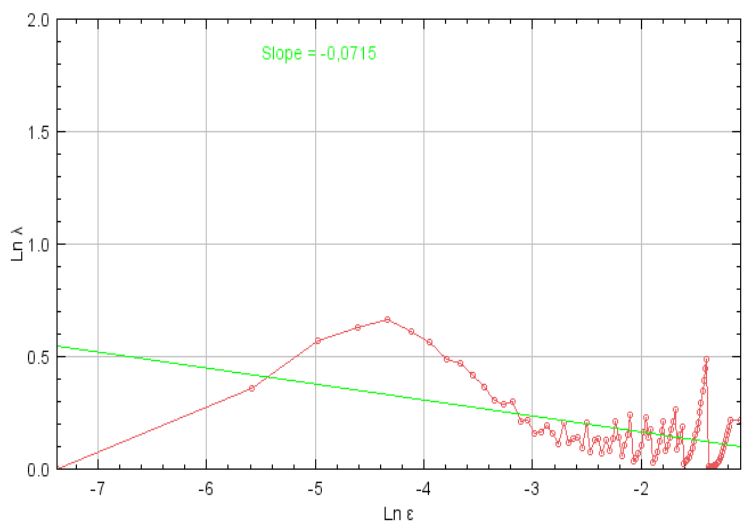

d)

Figure 5: The graphical representation of separate stages of lacunarity determining (non-uniformity of the graphite phase distribution in the volume of cast iron): a) GCl with laminar graphite; b) CIVG; c) DCl with spherical graphite No. 1; d) DCl with spherical graphite No. 2 
The calculation results of the dimensional and topological parameters of graphitized cast iron of different types

\begin{tabular}{ccccc}
\hline Cast iron & \multicolumn{4}{c}{ Parameter of graphite inclusions } \\
\hline & Shape $\left(\mathrm{D}_{\beta}\right)$ & Size $\left(\mathrm{A}_{\mathrm{gr}}\right)$ & Quantity $\left(\mathrm{S}_{\mathrm{gr}}\right)$ & Distribution $(\Lambda)$ \\
\hline $\mathrm{GCl}$ & 1.61 & 132.526 & 6.661 & 0.28 \\
$\mathrm{CIVG}$ & 1.69 & 198.653 & 14.283 & 0.28 \\
$\mathrm{DCl} \mathrm{No.} \mathrm{1}$ & 1.70 & 282.865 & 15.410 & 0.33 \\
$\mathrm{DCl} \mathrm{No.} \mathrm{2}$ & 1.71 & 203.998 & 16.585 & 0.30 \\
\hline
\end{tabular}

The results presented in the table have numerical values, so they can be used to construct mathematical models for evaluating the correlation between the structure and properties of graphitized cast iron.

\section{Conclusion}

The fractal analysis method makes it possible to solve the problems of identifying and determining the morphological parameters of different phases not only in metal alloys, but also in composite and nano- materials. The method can be used both for making a total analysis of the image and for determining such parameters as the shape and distribution of separate structural components and phases that is impossible by standard methods. In addition to all the advantages of the method it has got a number of shortcomings:

- the value $\mathrm{F}$ does not always give the smallest number of blocks with the side $\varepsilon$;

- the results of the fractal analysis method strongly depend both on the external action on the research objects and on the internal "content" of the images analysed.

Thus, at least two directions for further development of the method of fractal analysis of images of material structures can be suggested. The first implies taking into account not only the fractal dimension, but other fractal and non-fractal characteristics in the analysis. This will allow developing new methods, making the influence on the results minimal or completely null. The second direction is the development of new reference patterns, as well as methods and approaches for interpreting and evaluating results, since some operating standards and GOSTs are obsolescent and cannot satisfy the present-day high research requirements and new approaches being developed.

\section{References}

[1] A. G. Anisovich, I. N. Rumyantseva, The Practice of Metallographic Research of Materials, 2013.

[2] E. V. Timchenko, Digital Optical Microscopy, Samara, 2015.

[3] A. G. Anisovich, The art of metallography: application of optical staining methods, Vesti NAS of Belarus. 1 (2016) 36-42.

[4] A. G. Anisovich, The use of polarized light in the analysis of metals and alloys, Foundry Production and Metallurgy 3(67) (2012) 146-151.

[5] A. G. Anisovich, I. N. Rumyantseva, Visualization of the surface by differential interference contrast method, Foundry Production and Metallurgy 3(72) (2013) 156-162.

[6] V. G. Panteleev, O. V. Egorova, E. I. Klykova, Computer Microscopy, Moscow, 2005.

[7] Yu. V. Kuts, A. Yu. Povstyanoy, Modern methods of microstructure study with the applied programs via computer materials science, Scientific Notes 45 (2014) 323-329.

[8] A. G. Anisovich, I. N. Rumyantseva, L. V. Bisluk, Determination of the steel grain grade by computer methods, Foundry Production and Metallurgy 3 (2010) 100-104.

[9] A. G. Anisovich, A. V. Basalaj Assessment of operator's mistakes at quantitative analysis of the structure by computer methods, Foundry Production and Metallurgy 4(68) (2012) 145-150.

[10] J. Serra, Image Analysis and Mathematical Morphology, London, 1992. 
[11] O. Yu. Povstyanoy, V. A. Sichuk, V. D. Rud, O. V. Zabolotny, Morphological description, analysis and image processing of the microstructure of nozzles for sandblasting, produced by powder metallurgy methods, Scientific Notes 41 (2013) 203-210.

[12] K.V. Mardia, T.J. Hainsworth, A spatial thresholding method for image segmentation, IEEE Trans. Pattern Anal. Mach. Intell. 10 (1988) 919-927.

[13] N. V. Latypova, Fractal Analysis, Izhevsk, 2020.

[14] B. Mandelbrot, The Fractal Geometry of Nature, Moscow, 2002.

[15] O. V. Sotsenko, Computer DLA-model of the formation of spherical graphite in ductile cast iron, Metal and Casting of Ukraine 9 (2009) 3-9.

[16] B. A. Krylov, Fractal analysis of halftone images, Scientific and Technical Journal of Information Technologies, Mechanics and Optics 11 (2003) 112-114.

[17] D. G. Privezentsev, A. L. Zhiznyakov, Review of fractal methods of digital image processing in metallography, Algorithms, Methods and Systems of Data Processing 3 (2011) 133-141.

[18] A. L. Konyukhov, Guide to the application of ImageJ software package for image processing, 2012. URL: http://tu.tusur.ru/upload/posobia/ImageJ.pdf.

[19] K. V. Makarenko, D. A. Ilyushkin, Fractal analysis of microstructures of graphitized cast iron, Bulletin of Bryansk State Technical University 1 (2016) 34-43. 